\title{
Two Kinds of Nonlinear Matrix Equations and their Corresponding Matrix Sequences
}

\author{
Xiquan Shi, Fengshan Liu, Hanson Umoh, and Frank Gibson
}

\begin{abstract}
We studied the existence and uniqueness of solutions of the following two kinds of nonlinear matrix equations $X=\sum_{l=0}^{k-1} P_{l}^{T} X^{\alpha_{l}} P_{l}$, and $X=$ $\sum_{l=0}^{k-1}\left(P_{l}^{T} X P_{l}\right)^{\alpha_{l}}$, and the convergence of the matrix sequences $\left\{X_{i}\right\}$ recursively defined by $X_{n+k}=\sum_{l=0}^{k-1} P_{l}^{T} X_{n+l}^{\alpha_{l}} P_{l}, n \geq 0$, and $X_{n+k}=$ $\sum_{l=0}^{k-1}\left(P_{l}^{T} X_{n+l} P_{l}\right)^{\alpha_{l}}, n \geq 0$, where $\left|\alpha_{l}\right| \leq 1$ for $0 \leq l \leq k-1$ and $X_{0}, \cdots, X_{k-1}$ are initial positive definite matrices.
\end{abstract}

AMS classification: 40A05; 15A24

Keywords: Matrix; Positive definite matrix; Recursively defined sequence; Nonlinear equation

\section{Introduction}

Let $\mathbb{R}^{s}$ be the Euclidean $s$-space. A symmetric matrix $M$ is called positive definite or positive semidefinite if $\mathbf{v}^{T} M \mathbf{v}>0$ or $\mathbf{v}^{T} M \mathbf{v} \geq 0$ holds for any column vector $0 \neq \mathbf{v}=\left(v_{1}, \cdots, v_{s}\right)^{T} \in \mathbb{R}^{s}$, where $\mathbf{v}^{T}$ means the transpose of $\mathbf{v}$. It is easy to show that a diagonal matrix is positive definite or semidefinite if and only if each diagonal element is positive or non-negative respectively. For any $m \times n$ matrix $M$, the Moore-Penrose inverse(see [1]) $M^{\dagger}$ of $M$ is a $n \times m$ matrix satisfying the following equations,

$$
\begin{aligned}
& M M^{\dagger} M=M, \\
& M^{\dagger} M M^{\dagger}=M^{\dagger}, \\
& \left(M^{\dagger} M\right)^{T}=M^{\dagger} M, \\
& \left(M M^{\dagger}\right)^{T}=M M^{\dagger} .
\end{aligned}
$$

The Moore-Penrose inverse is a generalized inverse which exists and is unique for any matrix $M$. For a square matrix $M$ and $\alpha<0$, we define $M^{\alpha}=\left(M^{\dagger}\right)^{|\alpha|}$. Let $\Lambda=\operatorname{diag}\left(\lambda_{1}, \cdots, \lambda_{s}\right)$ be a diagonal matrix. Then its Moore-Penrose inverse is $\Lambda^{\dagger}=\operatorname{diag}\left(\lambda_{1}^{\dagger}, \cdots, \lambda_{s}^{\dagger}\right)$, where $\lambda_{i}^{\dagger}=\lambda_{i}^{-1}$ if $\lambda_{i} \neq 0$, and $\lambda_{i}^{\dagger}=0$ otherwise. For a symmetric matrix $M$, it is well known that there exist a diagonal matrix $\Lambda$ and an orthogonal matrix $Q$ such that $M=Q \Lambda Q^{T}$. It is easy to show that $M>0$ or $M \geq 0$ if and only if $\Lambda>0$ or $\Lambda \geq 0$ respectively. For a real number $\alpha$ and 
positive semidefinite matrix $M$, we define $M^{\alpha}=Q \Lambda^{\alpha} Q^{T}$ if $\alpha \geq 0$, where $\Lambda^{\alpha}=\operatorname{diag}\left(\lambda_{1}^{\alpha}, \cdots, \lambda_{s}^{\alpha}\right)$. Then $M^{\alpha}=\left(M^{\dagger}\right)^{|\alpha|}=Q\left(\Lambda^{\dagger}\right)^{|\alpha|} Q^{T}$ if $\alpha<0$.

Let $k$ be a counting number, let $P_{l}, 0 \leq l \leq k-1$ be $s \times s$ real matrices, and let $\alpha_{l}, 0 \leq l \leq k-1$ be real numbers. We studied the existence and uniqueness of solutions of the following two kinds of nonlinear equations

$$
X=\sum_{l=0}^{k-1} P_{l}^{T} X^{\alpha_{l}} P_{l},
$$

and

$$
X=\sum_{l=0}^{k-1}\left(P_{l}^{T} X P_{l}\right)^{\alpha_{l}},
$$

and the convergence of the matrix sequences $\left\{X_{i}\right\}$ recursively defined by

$$
X_{n+k}=\sum_{l=0}^{k-1} P_{l}^{T} X_{n+l}^{\alpha_{l}} P_{l}, n \geq 0,
$$

and

$$
X_{n+k}=\sum_{l=0}^{k-1}\left(P_{l}^{T} X_{n+l} P_{l}\right)^{\alpha_{l}}, n \geq 0,
$$

where the positive definite matrices $X_{0}, \cdots, X_{k-1}$ are initial matrices.

Let $\|\mathbf{v}\|$ denote the Euclidean norm of a vector $\mathbf{v} \in \mathbb{R}^{s}$. Let us view a $s \times s$ matrix $A=\left(a_{i, j}\right)_{1 \leq i, j \leq s}$ as a point in $\mathbb{R}^{s \times s}$, the Euclidean $s \times s$-space and define $\|A\|_{2}=\max _{\|\mathbf{v}\|=1}\|A \mathbf{v}\|$ to be the spectral norm of the matrix $A$.

Note 1.1 Note that all norms on a finite dimensional linear space are equivalent. Therefore, the results in this paper are true for any other norms.

Note 1.2 All the results in this paper are also right for complex matrices if we substitute transpose of, symmetric, and orthogonal matrices by conjugate of, Hermitian, and unitary matrices, respectively.

In [2] it is conjectured that the sequence of real numbers $A_{n}$ recursively defined by $A_{n+2}=\sqrt{A_{n+1}}+\sqrt{A_{n}}$ converges. In [4] this conjecture is proved. In this paper, we generalize sequences of numbers discussed in [4] into sequences of matrices. More precisely, we consider the following questions:

- Whether nontrivial $s \times s$ matrix solutions of the equation (2) exist? It is obvious that for any $\alpha_{l}, 0 \leq l \leq k-1, X=0$, the zero $s \times s$ matrix, is the trivial solution of (2).

- Is the nontrivial solution unique?

- Does the limit of the sequence $X_{i}$ defined by equation (4) exist? 
- What is the limit of the sequence $X_{i}$ ?

In section 2, we will prove the existence of a nontrivial positive semidefinite matrix solution of equation (2) if $\left|\alpha_{l}\right|<1,0 \leq l \leq k-1$. In section 3 , we study the corresponding recursively defined matrix sequence. We also prove that the matrix sequence is convergent to the solution of the corresponding nonlinear equation if $\left|\alpha_{l}\right|<1,0 \leq l \leq k-1$. As a consequence, we obtain that the nontrivial solution of (2) is unique. At the same time, we present a method to obtain the numerical solution of (2) under the conditions $\left|\alpha_{l}\right|<1,0 \leq l \leq k-1$. In section 4 , we will study other kinds of equations (3) and the corresponding matrix sequences (5).

\section{Nonlinear Matrix Equations}

First, we study the existence of positive definite matrix solutions of the equation (2), where

$$
\left|\alpha_{l}\right|<1,0 \leq l \leq k-1
$$

Theorem 2.1 Equation (2) has unique positive definite matrix solution if the matrix $C=\sum_{l=0}^{k-1} P_{l}^{T} P_{l}$ is positive definite.

The uniqueness will be given out in Section 3. To prove Theorem 2.1, we need the following Brouwer's Fixed-Point Theorem [5].

Lemma 2.1 Every continuous mapping $f: D \rightarrow D$ of a compact, convex, and nonempty set in $\mathbb{R}^{s}$ has a fixed point.

Let $\mathbb{R}_{+}=\left\{X \in \mathbb{R}^{s \times s} ; X\right.$ be a positive definite $s \times s$ matrix $\}$. It is easy to show that if both $X_{1}$ and $X_{2}$ are positive definite matrices, then (1$t) X_{1}+t X_{2}$ is also a positive definite matrix for $0 \leq t \leq 1$, i.e., $\mathbb{R}_{+}$is a convex set of $\mathbb{R}^{s \times s}$. For $s \times s$ matrices $P_{l}$ and real numbers $\alpha_{l}, 0 \leq l \leq k-1$, we define a mapping by

$$
f(X)=\sum_{l=0}^{k-1} P_{l}^{T} X^{\alpha_{l}} P_{l}, \quad X \in \mathbb{R}_{+} .
$$

The following lemma shows that if $C=\sum_{l=0}^{k-1} P_{l}^{T} P_{l}$ is positive definite, then $f$ is continuous from $\mathbb{R}_{+}$to itself. We first introduce the following notations:

$$
\begin{aligned}
\alpha & =\max \left\{\left|\alpha_{i}\right|, 0 \leq i \leq k-1\right\} \\
b & =\min \left\{\lambda_{\min }, \lambda_{\max }^{-1}\right\}, \\
B & =\max \left\{\lambda_{\max }, \lambda_{\min }^{-1}\right\}
\end{aligned}
$$

where $\lambda_{\min }$ and $\lambda_{\max }$ are the smallest and largest eigenvalues of the positive definite matrix $C=\sum_{l=0}^{k-1} P_{l}^{T} P_{l}$, respectively. It is easy to show 
that

$$
b=B^{-1} \leq 1 .
$$

Lemma 2.2 If $C=\sum_{l=0}^{k-1} P_{l}^{T} P_{l}$ is positive definite, then the mapping $f$, defined in (7), is continuous and from $\mathbb{R}_{+}$to itself.

Proof. First, we will show that $f$ maps $\mathbb{R}_{+}$into $\mathbb{R}_{+}$. For each $X \in \mathbb{R}_{+}$, let $\delta$ be a positive number smaller than both the minimum eigenvalue of $X$ and the reciprocal of the maximum eigenvalue of $X$. Then $X \geq \delta I$ and

$$
\begin{aligned}
f(X)=\sum_{l=0}^{k-1} P_{l}^{T} X^{\alpha_{l}} P_{l} & \geq \sum_{l=0}^{k-1} P_{l}^{T}(\delta I)^{\left|\alpha_{l}\right|} P_{l} \\
& \geq \sum_{l=0}^{k-1} P_{l}^{T}(\delta I)^{\alpha} P_{l}=\delta^{\alpha} C,
\end{aligned}
$$

where $I$ is the identity matrix. Therefore, $f$ maps $\mathbb{R}_{+}$into $\mathbb{R}_{+}$. The continuity of $f$ in (7) follows directly from Lemma 6.2.37 in [3].

We will use the Brouwer's Fixed Point Theorem on the mapping $f$. However $\mathbb{R}_{+}^{s}$ is not compact. To find a compact subset, for any positive number $a$, we set

$$
E_{a}=a^{\frac{1}{1-\alpha}} I .
$$

Let

$$
D=\left\{X \in \mathbb{R}_{+} ; E_{b} \leq X \leq E_{B}\right\} .
$$

Then we have the following lemma.

Lemma 2.3 $D$ is a closed, bounded, and convex set in $\mathbb{R}_{+}$. Furthermore, the mapping $f$ defined by (7) maps $D$ into $D$ if $C=\sum_{l=0}^{k-1} P_{l}^{T} P_{l}$ is positive definite, i.e.,

$$
f: D \rightarrow D \text {. }
$$

Proof. First, it is obvious and well known that $D$ is closed, bounded, and convex.

To show that $f$ maps $D$ into $D$, we first prove that if $X \in D$, then

$$
f(X) \geq E_{b}
$$

In fact, for $X \in D$ and each $l$, from the inequalities (8) and (9), we obtain that

$$
P_{l}^{T} X^{\alpha_{l}} P_{l} \geq P_{l}^{T}\left(b^{\frac{1}{1-\alpha}} I\right)^{\left|\alpha_{l}\right|} P_{l} \geq b^{\frac{\alpha}{1-\alpha}} P_{l}^{T} P_{l} .
$$

Therefore, we have

$$
\begin{aligned}
f(X) & =\sum_{l=0}^{k-1} P_{l}^{T} X^{\alpha_{l}} P_{l} \geq \sum_{l=0}^{k-1} b^{\frac{\alpha}{1-\alpha}} P_{l}^{T} P_{l}=b^{\frac{\alpha}{1-\alpha}} C \\
& \geq b^{\frac{\alpha}{1-\alpha}} b I=b^{\frac{1}{1-\alpha}} I=E_{b},
\end{aligned}
$$


i.e., inequality (10) holds. Similarly, since $B=b^{-1} \geq 1$, we have

$$
P_{l}^{T} X^{\alpha_{l}} P_{l} \leq P_{l}^{T}\left(B^{\frac{1}{1-\alpha}} I\right)^{\left|\alpha_{l}\right|} P_{l} \leq B^{\frac{\alpha}{1-\alpha}} P_{l}^{T} P_{l}
$$

and

$$
\begin{aligned}
f(X) & =\sum_{l=0}^{k-1} P_{l}^{T} X^{\alpha_{l}} P_{l} \leq \sum_{l=0}^{k-1} B^{\frac{\alpha}{1-\alpha}} \sum_{l=0}^{k-1} P_{l}^{T} P_{l} \\
& =B^{\frac{\alpha}{1-\alpha}} C \leq B^{\frac{\alpha}{1-\alpha}} B I=B^{\frac{1}{1-\alpha}} I=E_{B},
\end{aligned}
$$

i.e., $f(X) \leq E_{B}$. So $f(X) \in D$, i.e., $f$ maps $D$ into $D$. Lemma 2.3 is proved

Proof of Theorem 2.1. From Lemma 2.2 and Lemma 2.3, the assumptions of Lemma 2.1 (Brouwer's Fixed-Point Theorem) are satisfied. Therefore, the mapping $f$ has a fixed point in $D$. A fixed point of $f$ in $D$ is a solution of equation (2) in $D$. Since every matrix in $D$ is positive definite, the solution of equation (2) in $D$ is positive definite. Thus we proved the Theorem 2.1

Theorem 2.1 indicates that if $C=\sum_{l=0}^{k-1} P_{l}^{T} P_{l}$ is positive definite, then the equation (2) has a positive definite solution. Next, we consider the condition that $C$ be positive semidefinite rather than positive definite.

Theorem 2.2 Equation (2) has a positive semidefinite but not positive definite matrix solution if $C=\sum_{l=0}^{k-1} P_{l}^{T} P_{l}$ is a positive semidefinite matrix, but not a positive definite matrix.

Non-trivial solutions of (2) exist under assumptions in Theorem 2.2 except some special cases which will be explained in a later theorem.

Proof of Theorem 2.2. The proof is by mathematical induction on $s$, the order of the matrix $C$. If $s=1, P_{l}, 0 \leq l \leq k-1$ and $C$ are real numbers. Thus, $C=\sum_{l=0}^{k-1} P_{l}^{T} P_{l}$ being a positive semidefinite matrix, but not positive definite matrix means that $C=\sum_{l=0}^{k-1} P_{l}^{2}=0$, i.e., $P_{l}=0,0 \leq l \leq k-1$. This means (2) has only a trivial solution. Theorem 2.2 is therefore true. We assume that Theorem 2.2 is true for $s=t-1$. By the mathematical induction, we only need to show that the theorem is true for $s=t$. Since $C=\sum_{l=0}^{k-1} P_{l}^{T} P_{l}$ is positive semidefinite and not positive definite, then $C$ is singular. Therefore zero is an eigenvalue of $C$, i.e., there exists an unit eigenvector $\mathbf{v} \in \mathbb{R}^{t}$ such that $C \mathbf{v}=0$. Thus, we have

$$
\sum_{l=0}^{k-1}\left\|P_{l} \mathbf{v}\right\|^{2}=\sum_{l=0}^{k-1}\left(P_{l} \mathbf{v}\right)^{T} P_{l} \mathbf{v}=\sum_{l=0}^{k-1} \mathbf{v}^{T} P_{l}^{T} P_{l} \mathbf{v}=\mathbf{v}^{T} C \mathbf{v}=0 .
$$

This means that

$$
P_{l} \mathbf{v}=0, \quad 0 \leq l \leq k-1
$$


i.e., $\mathbf{v}$ is a common unit eigenvector of $P_{l}, 0 \leq l \leq k-1$. Thus, there exist column vectors $\mathbf{v}_{1}=\mathbf{v}, \mathbf{v}_{2}, \cdots, \mathbf{v}_{t}$ such that $Q=\left(\mathbf{v}_{1} \mathbf{v}_{2} \cdots \mathbf{v}_{t}\right)$ is an orthogonal matrix and

$$
P_{l}=Q \widehat{P}_{l} Q^{T}, 0 \leq l \leq k-1,
$$

where

$$
\widehat{P}_{l}=\left(\begin{array}{cc}
0 & P_{l}^{*} \\
0 & \bar{P}_{l}
\end{array}\right),
$$

and $P_{l}^{*}$ is a $(t-1)$-row vector and $\bar{P}_{l}$ is a $(t-1) \times(t-1)$ matrix. By denoting $\widehat{X}=Q^{T} X Q$, in (11), equation (2) is equivalent to

$$
\widehat{X}=\sum_{l=0}^{k-1} \widehat{P}_{l}^{T} \widehat{X}^{\alpha_{l}} \widehat{P}_{l} .
$$

If $\bar{C}=\sum_{l=0}^{k-1} \bar{P}_{l}^{T} \bar{P}_{l}$ is positive definite, then, according to Theorem 2.1,

$$
\bar{X}=\sum_{l=0}^{k-1} \bar{P}_{l}^{T} \bar{X}^{\alpha_{l}} \bar{P}_{l},
$$

has a positive definite matrix solution. If $\bar{C}=\sum_{l=0}^{k-1} \bar{P}_{l}^{T} \bar{P}_{l}$ is positive semidefinite, but not positive definite, then according to the mathematical induction, (13) also has a positive semidefinite matrix solution. If $\bar{X}$ is a solution of (13), it is easy to verify that

$$
\widehat{X}=\left(\begin{array}{ll}
0 & 0 \\
0 & \bar{X}
\end{array}\right)
$$

is a solution of (12). And $X=Q \widehat{X} Q^{T}$ is a positive semidefinite solution of (2).

In the following theorem, we will answer the question raised earlier about the existence of only trivial solution of (2).

Theorem 2.3 Equation (2) has only trivial solution $X=0$ if and only if there exist an orthogonal matrix $Q$ and upper-triangular matrices $U_{l}, 0 \leq$ $l \leq k-1$, with zero diagonal elements, i.e., all the diagonal elements of $U_{l}, 0 \leq l \leq k-1$, are zeros, such that $P_{l}=Q U_{l} Q^{T}, 0 \leq l \leq k-1$.

Proof. We use mathematical induction on $s$, the order of the matrix $C$, to prove Theorem 2.3. We first prove the necessity, i.e., if (2) has only a trivial solution, then there exist an orthogonal matrix $Q$ and uppertriangular matrices $U_{l}, 0 \leq l \leq k-1$, with zero diagonal elements, i.e., all the diagonal elements of $U_{l}, 0 \leq l \leq k-1$, are zeros, such that $P_{l}=Q U_{l} Q^{T}, 0 \leq l \leq k-1$. For $s=1$, we will show that if (2) has only 
a trivial solution, then $C=\sum_{l=0}^{k-1} P_{l}^{T} P_{l}=\sum_{l=0}^{k-1} P_{l}^{2}=0$. Otherwise, if $C \neq 0$, we consider the following function,

$$
h(x)=\sum_{l=0}^{k-1} P_{l}^{2} x^{\alpha_{l}-1}-1 .
$$

It is easy to show that $h(x)$ is strictly decreasing in the interval $(0, \infty)$, $\lim _{x \rightarrow 0^{+}} h(x)=\infty$, and $\lim _{x \rightarrow \infty} h(x)=-1$. There must exists $x>0$ such that $h(x)=0$. Note that a solution of $h(x)=0$ is also a solution of (2). This is a contradiction to our assumption that equation (2) has only a trivial solution. Thus, $P_{l}=0,0 \leq l \leq k-1$, if (2) has only a trivial solution. Now, we assume that the necessity is true for $s=t-1$. By mathematical induction, we only have to show that the necessity is true for $s=t$. Repeating the proof of Theorem 2.2, we have the existence of an orthogonal matrix $Q$ and matrices $\widehat{P}_{l}$ satisfying (11). And (13) has also only a trivial solution, otherwise $\widehat{X}$, defined by (14), will be a non-trivial solution of (12) if $\bar{X}$ is a non-trivial solution of (13). Thus, according to the induction, there exists a $(t-1) \times(t-1)$ orthogonal matrix $\bar{Q}$ such that $\bar{P}_{l}=\bar{Q} \bar{U}_{l} \bar{Q}^{T}, 0 \leq l \leq k-1$, where $\bar{U}_{l}$ 's are upper triangular matrices with zero diagonal elements. And

$$
\begin{aligned}
P_{l} & =Q \widehat{P}_{l} Q^{T}=Q\left(\begin{array}{ll}
0 & P_{l}^{*} \\
0 & \bar{P}_{l}
\end{array}\right) Q^{T} \\
& =Q\left(\begin{array}{ll}
1 & 0 \\
0 & \bar{Q}
\end{array}\right)\left(\begin{array}{ll}
0 & P_{l}^{*} \\
0 & \bar{U}_{l}
\end{array}\right)\left(\begin{array}{ll}
1 & 0 \\
0 & \bar{Q}^{T}
\end{array}\right) Q^{T}, 0 \leq l \leq k-1 .
\end{aligned}
$$

The necessity is proved.

We still use mathematical induction to prove the sufficiency of Theorem 2.3. For $s=1$, the fact that there exist an orthogonal matrix $Q$ and upper-triangular matrices $U_{l}, 0 \leq l \leq k-1$ with zero diagonal elements such that $P_{l}=Q U_{l} Q^{T}, 0 \leq l \leq k-1$ simply means $P_{l}=0,0 \leq l \leq k-1$. In this case, (2) has only a trivial solution. Assuming that the sufficiency is right for $s=t-1$, we will show that it is also true for $s=t$. Let $P_{l}=Q U_{l} Q^{T}, 0 \leq l \leq k-1$. If $X$ is a solution of (2), then $\widehat{X}=Q^{T} X Q$ is a solution of

$$
\widehat{X}=\sum_{l=0}^{k-1} U_{l}^{T} \widehat{X}^{\alpha_{l}} U_{l} .
$$

Since $U_{l}$ is upper triangular matrix, we have

$$
U_{l}=\left(\begin{array}{cc}
0 & \widetilde{U}_{l} \\
0 & \bar{U}_{l}
\end{array}\right), \quad 0 \leq l \leq k-1,
$$

where $\bar{U}_{l}$ is a $(t-1) \times(t-1)$ matrix and $\widetilde{U}_{l}$ is a $t-1$ row vector. It is easy to check that the elements on the first column and the first row of 
each matrix $U_{l}^{T} \widehat{X}^{\alpha_{l}} U_{l}$ must be all zeros, and so is $\widehat{X}$. Therefore we have the following form

$$
\widehat{X}=\left(\begin{array}{ll}
0 & 0 \\
0 & \bar{X}
\end{array}\right)
$$

where $\bar{X}$ is a $(t-1) \times(t-1)$ matrix. Combining (16), it yields

$$
\bar{X}=\sum_{l=0}^{k-1} \bar{U}_{l}^{T} \bar{X}^{\alpha_{l}} \bar{U}_{l}
$$

According to our assumption for $s=t-1$, the equation above has only a trivial solution. Theorem 2.3 is proved.

The solution of (2) is usually very difficulty to obtain and has no explicit form. The following theorem shows that under certain conditions, we can solve (2) more efficiently.

Theorem 2.4 If there exist an orthogonal matrix $Q$ and diagonal matrices $\Lambda_{l}=\operatorname{diag}\left(\lambda_{l, 1}, \cdots, \lambda_{l, s}\right), 0 \leq l \leq k-1$ such that $P_{l}=Q \Lambda_{l} Q^{T}, 0 \leq l \leq$ $k-1$, then $X=Q \Lambda Q^{T}$ is a solution of (2), where $\Lambda=\operatorname{diag}\left(\lambda_{1}, \cdots, \lambda_{s}\right)$ and $\lambda_{i}$ is the unique positive solution of

$$
\lambda_{i}=\sum_{l=0}^{k-1} \lambda_{l, i}^{2} \lambda_{i}^{\alpha_{l}}, 1 \leq i \leq s
$$

if $\sum_{l=0}^{k-1} \lambda_{l, i}^{2}>0$, and $\lambda_{i}=0$ if $\sum_{l=0}^{k-1} \lambda_{l, i}^{2}=0$.

Proof. If $\sum_{l=0}^{k-1} \lambda_{l, i}^{2}>0$, similar to (15), it is easy to prove $\sum_{l=0}^{k-1} \lambda_{l, i}^{2} \lambda^{\alpha_{l}-1}$ $1=0$ has a unique positive solution $\lambda_{i}$ which is also a solution of equation (17). If $\sum_{l=0}^{k-1} \lambda_{l, i}^{2}=0$, then $\lambda_{i}=0$. For $\lambda_{i}=0, \lambda_{i}^{\alpha}=\left(\lambda_{i}^{\dagger}\right)^{|\alpha|}=0$ if $\alpha<0$. Thus, $\sum_{l=0}^{k-1} \lambda_{l, i}^{2} \lambda_{i}^{\alpha_{l}}=0=\lambda_{i}$. Therefore, we have

$$
\sum_{l=0}^{k-1} \Lambda_{l} \Lambda^{\alpha_{l}} \Lambda_{l}=\operatorname{diag}\left(\sum_{l=0}^{k-1} \lambda_{l, 1}^{2} \lambda_{1}^{\alpha_{l}}, \cdots, \sum_{l=0}^{k-1} \lambda_{l, s}^{2} \lambda_{s}^{\alpha_{l}}\right)=\operatorname{diag}\left(\lambda_{1}, \cdots, \lambda_{s}\right) .
$$

Finally, it follows that

$$
\begin{aligned}
\sum_{l=0}^{k-1} P_{l}^{T} X^{\alpha_{l}} P_{l} & =\sum_{l=0}^{k-1} Q \Lambda_{l} Q^{T} X^{\alpha_{l}} Q \Lambda_{l} Q^{T} \\
& =Q \sum_{l=0}^{k-1} \Lambda_{l} \Lambda^{\alpha_{l}} \Lambda_{l} Q^{T}=Q \Lambda Q^{T}=X .
\end{aligned}
$$

This completes the proof of Theorem 2.4

In the next section, we will also show that the non-trivial solution of equation (2) in any of the Theorems of this section is unique as a consequence of the limit of a recursively defined sequence. 


\section{Recursively Defined Matrix Sequences}

Before discuss the uniqueness of the solution of (2), we consider the following recursively defined matrix sequence.

$$
X_{n+k}=\sum_{l=0}^{k-1} P_{l}^{T} X_{n+l}^{\alpha_{l}} P_{l}, n \geq 0,
$$

where $X_{0}, \cdots, X_{k-1}$ are initial positive definite matrices. For the matrix sequence $\left\{X_{i}: i \geq 1\right\}$ recursively defined by (18), we obtain the following theorem.

Theorem 3.1 If $C=\sum_{l=0}^{k-1} P_{l}^{T} P_{l}$ is positive definite, then for arbitrary initial positive definite matrices $X_{i}>0,0 \leq i \leq k-1$, the matrix sequence $\left\{X_{i}: i \geq 1\right\}$ defined by (18) converges to a positive definite matrix solution of (2), independent of the initial matrices $X_{i}>0,0 \leq i \leq k-1$. Moreover, the positive definite solution of (2) is unique, since the limit of the matrix sequence is unique.

Proof. According to Theorem 2.1, equation (2) has a positive definite matrix solution $X>0$. Therefore, there exist positive numbers $0<a_{0} \leq 1$ and $a_{1} \geq 1$ such that the initial matrices satisfy the inequality

$$
a_{0} X \leq X_{i} \leq a_{1} X, 0 \leq i \leq k-1
$$

since $X_{i}, 0 \leq i \leq k-1$ are positive definite matrices. In fact, we can take

$$
0<a_{0} \leq \min \left\{\min \left\{1, \frac{\lambda_{\min , i}}{\lambda_{\max }} ; 0 \leq i \leq k-1\right\}, \frac{1}{\max \left\{1, \frac{\lambda_{\max , i}}{\lambda_{\min }} ; 0 \leq i \leq k-1\right\}}\right\},
$$

and

$$
a_{1}=a_{0}^{-1}
$$

where $\lambda_{\min }$ and $\lambda_{\max }$ are the smallest and largest eigenvalues of matrix $X$, $\lambda_{\min , i}$ and $\lambda_{\max , i}, 0 \leq i \leq k-1$ are the smallest and largest eigenvalues of matrix $X_{i}$, respectively. Let $n=m k+r$, where $0 \leq r<k$ and $m$ is a nonnegative integer. We will prove the following inequalities,

$$
\left(a_{0}\right)^{\alpha^{m}} X \leq X_{n} \leq\left(a_{1}\right)^{\alpha^{m}} X, 0 \leq r<k
$$

where $\alpha$ is defined in equation (8). If we assume that inequality (20) holds, then it is easy to show that $\alpha^{m} \rightarrow 0,\left(a_{0}\right)^{\alpha^{m}} \rightarrow 1$ and $\left(a_{1}\right)^{\alpha^{m}} \rightarrow 1$ as $m \rightarrow \infty$, since $0 \leq \alpha<1$. Therefore,

$$
\lim _{n \rightarrow \infty} X_{n}=X
$$

This also means that the positive definite matrix solution of (2) is unique, since the limit of matrix sequence $\left\{X_{n}: n \geq 1\right\}$ is unique. To complete 
the proof of Theorem 3.1, we only need to prove the inequality (20). To this end, we will use mathematical induction on $m$. From inequality (19), inequality (20) holds for $m=0$. Assume that (20) is true for $m=p-1$, i.e., for $n=(p-1) k+r, 0 \leq r<k$,

$$
\left(a_{1}\right)^{\alpha^{p-1}} X \geq X_{n} \geq\left(a_{0}\right)^{\alpha^{p-1}} X, 0 \leq r<k
$$

holds. We only need to prove that (20) is true for $m=p$. If $\alpha_{l} \geq 0$, it is easy to check $X_{(p-1) k+l}^{\alpha_{l}} \geq\left(a_{0}\right)^{\alpha^{p-1} \alpha_{l}} X^{\alpha_{l}}$. If $\alpha_{l}<0$, then $X_{(p-1) k+l}^{\alpha_{l}} \geq$ $\left(\left(a_{1}\right)^{\alpha^{p-1}} X\right)^{\alpha_{l}}=\left(a_{0}\right)^{\alpha^{p-1}\left|\alpha_{l}\right|} X^{\alpha_{l}}$ since $a_{1}=a_{0}^{-1}$. Thus, from inequality (21), we have

$$
\begin{aligned}
X_{p k} & =\sum_{l=0}^{k-1} P_{l}^{T} X_{(p-1) k+l}^{\alpha_{l}} P_{l} \geq \sum_{l=0}^{k-1} P_{l}^{T}\left(a_{0}\right)^{\alpha^{p-1}\left|\alpha_{l}\right|} X^{\alpha_{l}} P_{l} \\
& =\sum_{l=0}^{k-1}\left(a_{0}\right)^{\left|\alpha_{l}\right| \alpha^{p-1}} P_{l}^{T} X^{\alpha_{l}} P_{l} \geq\left(a_{0}\right)^{\alpha^{p}} \sum_{l=0}^{k-1} P_{l}^{T} X^{\alpha_{l}} P_{l} \\
& =\left(a_{0}\right)^{\alpha^{p}} X,
\end{aligned}
$$

since $a_{0} \leq 1, \alpha_{l} \leq \alpha, 0 \leq l<k$, and $X$ is a positive definite matrix solution of (2). Furthermore, using $X_{p k} \geq\left(a_{0}\right)^{\alpha^{p}} X$, we obtain

$$
\begin{aligned}
X_{p k+1} & =\sum_{l=0}^{k-1} P_{l}^{T} X_{(p-1) k+l+1}^{\alpha_{l}} P_{l}=\sum_{l=0}^{k-2} P_{l}^{T} X_{(p-1) k+l+1}^{\alpha_{l}} P_{l}+P_{k-1}^{T} X_{p k}^{\alpha_{k-1}} P_{k-1} \\
& \geq \sum_{l=0}^{k-2} P_{l}^{T}\left(a_{0}\right)^{\alpha^{p-1}\left|\alpha_{l}\right|} X^{\alpha_{l}} P_{l}+P_{k-1}^{T} a_{0}^{\alpha^{p}\left|\alpha_{k-1}\right|} X^{\alpha_{k-1}} P_{k-1} \\
& =\sum_{l=0}^{k-2}\left(a_{0}\right)^{\left|\alpha_{l}\right| \alpha^{p-1}} P_{l}^{T} X^{\alpha_{l}} P_{l}+a_{0}^{\left|\alpha_{k-1}\right| \alpha^{p}} P_{k-1}^{T} X^{\alpha_{k-1}} P_{k-1} \\
& \geq\left(a_{0}\right)^{\alpha^{p}} \sum_{l=0}^{k-1} P_{l}^{T} X^{\alpha_{l}} P_{l} \\
& =\left(a_{0}\right)^{\alpha^{p}} X
\end{aligned}
$$

since $a_{0}^{\left|\alpha_{k-1}\right| \alpha^{p}} \geq a_{0}^{\alpha^{p}}$. Using $X_{p k} \geq\left(a_{0}\right)^{\alpha^{p}} X$ and $X_{p k+1} \geq\left(a_{0}\right)^{\alpha^{p}} X$, we can show that $X_{p k+2} \geq\left(a_{0}\right)^{\alpha^{p}} X$. Repeatedly using the same processes with the first $i$ inequalities $X_{p k+t} \geq\left(a_{0}\right)^{\alpha^{p}} X, 0 \leq t \leq i-1$ for $(i<k)$, we have

$$
X_{p k+i} \geq\left(a_{0}\right)^{\alpha^{p}} X, 2 \leq i<k .
$$

This means

$$
X_{p k+r} \geq\left(a_{0}\right)^{\alpha^{p}} X, 0 \leq r<k .
$$

Similarly, we can obtain

$$
X_{p k+r} \leq\left(a_{1}\right)^{\alpha^{p}} X, 0 \leq r<k .
$$

Therefore, we have proved inequality (20).

By Theorem 2.3, equation (2) has only a trivial solution $X=0$ if and only if there exist an orthogonal matrix $Q$ and upper-triangular matrices $U_{l}, 0 \leq l \leq k-1$ with zero diagonal elements such that $P_{l}=Q U_{l} Q^{T}, 0 \leq$ $l \leq k-1$. If $C=\sum_{l=0}^{k-1} P_{l}^{T} P_{l}$ is positive definite, then, according to 
Theorem 2.1, equation (2) has a positive definite solution. Furthermore, the matrix sequence defined by (18) converges to this solution. If $C$ is positive semidefinite, but not positive definite, from the proof of Theorem 2.3 , there exist $1 \leq \tau \leq s$ and an orthogonal matrix $Q$ such that

$$
\widehat{P}_{l}=\left(\begin{array}{cc}
U_{l} & V_{l} \\
0 & \bar{P}_{l}
\end{array}\right), \quad 0 \leq l \leq k-1
$$

and $P_{l}=Q \widehat{P}_{l} Q^{T}, 0 \leq l \leq k-1$, where each $U_{l}$ is a $\tau \times \tau$ upper triangular matrix with zero diagonal elements, each $V_{l}$ is a $\tau \times(s-\tau)$ matrix, and $\bar{C}=\sum_{l=0}^{k-1} \bar{P}_{l}^{T} \bar{P}_{l}$ is a positive definite matrix. In case of $\tau=s, \bar{P}_{l}$ and $V_{l}$ will not appear and $\widehat{P}_{l}=U_{l}$, and according to Theorem 2.3, equation (2) has only a trivial solution. Therefore, in the following, we always assume that $1 \leq \tau<s$. We consider the following matrix sequence

$$
\widehat{X}_{n+k}=\sum_{l=0}^{k-1} \widehat{P}_{l}^{T} \widehat{X}_{n+l}^{\alpha_{l}} \widehat{P}_{l}, n \geq 0,
$$

where $\widehat{X}_{0}, \cdots, \widehat{X}_{k-1}$ are initial positive definite matrices. For the matrix sequence $\left\{\widehat{X}_{i}: i \geq 1\right\}$ recursively defined by (22), we obtain the following theorem.

Theorem 3.2 For arbitrary initial positive definite matrices $\widehat{X}_{i}>0,0 \leq$ $i \leq k-1$, the matrix sequence $\left\{\widehat{X}_{i}: i \geq 1\right\}$ defined by (22) converges to

$$
\widehat{X}=\left(\begin{array}{ll}
0 & 0 \\
0 & \bar{X}
\end{array}\right)
$$

which is independent of the initial matrices $\widehat{X}_{i}>0,0 \leq i \leq k-1$, if $\bar{C}=\sum_{l=0}^{k-1} \bar{P}_{l}^{T} \bar{P}_{l}$ is a positive definite matrix, where $\bar{X}$ is a positive definite $(s-\tau) \times(s-\tau)$ matrix solution of the following equation.

$$
\bar{X}=\sum_{l=0}^{k-1} \bar{P}_{l}^{T} \bar{X}^{\alpha_{l}} \bar{P}_{l} .
$$

Lemma 3.1 For $i \geq m k, 1 \leq m \leq \tau$,

$$
\widehat{X}_{i}=\left(\begin{array}{cc}
0 & 0 \\
0 & \bar{X}_{i}
\end{array}\right),
$$

where each $\bar{X}_{i}$ is a $(s-m) \times(s-m)$ matrix.

Proof. It is easy to check that all elements in the first column and the first row of $\widehat{P}_{l}^{T} \widehat{X}_{i-k+l}^{\alpha_{l}} \widehat{P}_{l}, 0 \leq l<k, i \geq k$ are zeros, and so is $\widehat{X}_{i}=$ $\sum_{l=0}^{k-1} \widehat{P}_{l}^{T} \widehat{X}_{i-k+l}^{\alpha_{l}} \widehat{P}_{l}$. Assume the elements in the first $h-1,1 \leq h \leq \tau$, rows and the first $h-1$ columns of $\widehat{X}_{i}$ are all zeros for $i \geq(h-1) k$. We 
will prove that the elements in the first $h$ rows and the first $h$ columns of $\widehat{X}_{i}$ are also all zeros for $i \geq h k$. Let $i=n+k$ and $n \geq(h-1) k$. Since

$$
\widehat{X}_{n+k}=\sum_{l=0}^{k-1} \widehat{P}_{l}^{T} \widehat{X}_{n+l}^{\alpha_{l}} \widehat{P}_{l}, n \geq 0,
$$

we only need to focus on $\widehat{P}_{l}^{T} \widehat{X}_{n+l}^{\alpha_{l}} \widehat{P}_{l}$ for each $l$. Since $n+l \geq(h-1) k$, we have

$$
\widehat{X}_{n+l}^{\alpha_{l}}=\left(\begin{array}{cc}
0 & 0 \\
0 & \bar{X}_{n+l}
\end{array}\right)^{\alpha_{l}}=\left(\begin{array}{cc}
0 & 0 \\
0 & \bar{X}_{n+l}^{\alpha_{l}}
\end{array}\right),
$$

where $\bar{X}_{n+l}$ is a $(s-h+1) \times(s-h+1)$ matrix. We denote

$$
\widehat{P}_{l}=\left(\begin{array}{cc}
\tilde{U}_{l} & \tilde{V}_{l} \\
0 & \tilde{P}_{l}
\end{array}\right)
$$

where $\tilde{P}_{l}$ is a $(s-h+1) \times(s-h+1)$ matrix, and the elements in the first column of $\tilde{P}_{l}$ are all zeros. Thus,

$$
\begin{aligned}
& \widehat{P}_{l}^{T} \widehat{X}_{n+l}^{\alpha_{l}} \widehat{P}_{l}=\left(\begin{array}{cc}
\tilde{U}_{l}^{T} & 0 \\
\tilde{V}_{l}^{T} & \tilde{P}_{l}^{T}
\end{array}\right)\left(\begin{array}{cc}
0 & 0 \\
0 & \tilde{X}_{n+l}
\end{array}\right)\left(\begin{array}{cc}
\tilde{U}_{l} & \tilde{V}_{l} \\
0 & \tilde{P}_{l}
\end{array}\right) \\
& =\left(\begin{array}{cc}
0 & 0 \\
0 & \tilde{P}_{l}^{T} \tilde{X}_{n+l} \tilde{P}_{l}
\end{array}\right) \text {. }
\end{aligned}
$$

Since the elements in the first column of $\tilde{P}_{l}$ are all zeros, it is easy to check that the elements in the first row and the first column of $\tilde{P}_{l}^{T} \tilde{X}_{n+l} \tilde{P}_{l}$ are all zeros, i.e., the elements in the first $h$ rows and the first $h$ columns of $\widehat{X}_{i}=\widehat{X}_{n+k}$ are zeros for $i \geq h k$. By mathematical induction, we have that the elements in the first $\tau$ rows and the first $\tau$ columns of $\widehat{X}_{i}$ are all zeros, i.e., for $i \geq \tau k$, we have

$$
\widehat{X}_{i}=\left(\begin{array}{cc}
0 & 0 \\
0 & \bar{X}_{i}
\end{array}\right)
$$

where each $\bar{X}_{i}$ is a $(s-\tau) \times(s-\tau)$ matrix.

Proof of Theorem 3.2. According to Lemma 3.1, for $n \geq \tau k$, we have

$$
\begin{aligned}
\left(\begin{array}{cc}
0 & 0 \\
0 & \bar{X}_{n+k}
\end{array}\right) & =\widehat{X}_{n+k}=\sum_{l=0}^{k-1} \widehat{P}_{l}^{T} \widehat{X}_{n+l}^{\alpha_{l}} \widehat{P}_{l} \\
& =\sum_{l=0}^{k-1}\left(\begin{array}{cc}
U_{l}^{T} & 0 \\
V_{l}^{T} & \bar{P}^{T}
\end{array}\right)\left(\begin{array}{cc}
0 & 0 \\
0 & \bar{X}_{n+l}
\end{array}\right){ }^{\alpha_{l}}\left(\begin{array}{cc}
U_{l} & V_{l} \\
0 & \bar{P}
\end{array}\right) \\
& =\sum_{l=0}^{k-1}\left(\begin{array}{cc}
U_{l}^{T} & 0 \\
V_{l}^{T} & \bar{P}^{T}
\end{array}\right)\left(\begin{array}{cc}
0 & 0 \\
0 & \bar{X}_{n+l}^{\alpha_{l}}
\end{array}\right)\left(\begin{array}{cc}
U_{l} & V_{l} \\
0 & \bar{P}
\end{array}\right) \\
& =\left(\begin{array}{cc}
0 & 0 \\
0 & \sum_{l=0}^{k-1} \bar{P}^{T} \bar{X}_{n+l}^{\alpha_{l}} \bar{P}
\end{array}\right) .
\end{aligned}
$$


Thus,

$$
\bar{X}_{n+k}=\sum_{l=0}^{k-1} \bar{P}^{T} \bar{X}_{n+l}^{\alpha_{l}} \bar{P}, n \geq \tau k .
$$

Since $\bar{C}=\sum_{l=0}^{k-1} \bar{P}^{T} \bar{P}$ is positive definite, according to Theorem 3.1, the matrix sequence $\left\{\bar{X}_{n}\right\}$ defined in (24) converge to $\bar{X}$, the positive definite matrix solution of (23), independent of the initial matrices $\widehat{X}_{i}>0,0 \leq$ $i \leq k-1$. According to Lemma 3.1, for $i \geq \tau k$, the matrix sequence

$$
\widehat{X}_{i}=\left(\begin{array}{cc}
0 & 0 \\
0 & \bar{X}_{i}
\end{array}\right),
$$

defined by (22), converges to

$$
\widehat{X}=\left(\begin{array}{cc}
0 & 0 \\
0 & \bar{X}
\end{array}\right)
$$

independent of the initial matrices $\widehat{X}_{i}>0,0 \leq i \leq k-1$. Theorem 3.2 is proved

With Theorem 3.2, it is easy to obtain the following corollary.

Corollary 3.1 For arbitrary initial positive definite matrices $X_{i}>0,0 \leq$ $i \leq k-1$, the matrix sequence $\left\{X_{i}: i \geq 1\right\}$ defined by (18) converges to

$$
X=Q\left(\begin{array}{cc}
0 & 0 \\
0 & \bar{X}
\end{array}\right) Q^{T}
$$

which is independent of the initial matrices $X_{i}>0,0 \leq i \leq k-1$, where $\bar{X}$ is the unique positive definite matrix solution of (23).

Since the limit of a matrix sequence $\left\{X_{n}\right\}$ is unique, the nonnegative and nontrivial solution of the equation (2) in Theorem 2.1 and Theorem 2.2 is unique.

In Theorem 3.1, Theorem 3.2, and Corollary 3.1, we require that the initial matrices are positive definite. We know that for some square matrix $X$ and real number $\alpha, X^{\alpha}$ may have no definition. Therefore, we could not obtain a matrix sequence by (2) if we don't ask the initial matrices symmetric. However, it is very interesting that the sequence $\left\{X_{i}: i \geq 1\right\}$ defined by (18) always converges to the positive definite solution of (2) for random initial matrices when we do simulations with computers. 


\section{Another Kind of Nonlinear Equations and Recursively Defined Matrix Sequences}

In this section, we study, under the same assumption of equation (2), the existence of positive solutions of the following equation

$$
X=\sum_{l=0}^{k-1}\left(P_{l}^{T} X P_{l}\right)^{\alpha_{l}}, X \in \mathbb{R}_{+},
$$

and recursively defined vector sequence

$$
X_{n+k}=\sum_{l=0}^{k-1}\left(P_{l}^{T} X_{n+l} P_{l}\right)^{\alpha_{l}}, n \geq 0,
$$

Since all proofs and results are parallel to those for equation (2) and sequence (18), we only give out some main conclusions for equation (25) and sequence (26).

Theorem 4.1 Equation (25) has unique positive definite matrix solution if the matrices $C=\sum_{l=0}^{k-1}\left(P_{l}^{T} P_{l}\right)^{\alpha_{l}}$ is positive definite.

Theorem 4.2 Equation (25) has a positive semidefinite matrix solution if $C=\sum_{l=0}^{k-1}\left(P_{l}^{T} P_{l}\right)^{\alpha_{l}}$ is a positive semidefinite matrix, but not a positive definite matrix.

Theorem 4.3 Equation (2) has only trivial solution $X=0$ if and only if there exist an orthogonal matrix $Q$ and upper-triangular matrices $U_{l}, 0 \leq$ $l \leq k-1$ with zero diagonal elements, i.e., all the diagonal elements of $U_{l}, 0 \leq l \leq k-1$ are zeros, such that $P_{l}=Q U_{l} Q^{T}, 0 \leq l \leq k-1$.

Theorem 4.4 If there exist an orthogonal matrix $Q$ and diagonal matrices $\Lambda_{l}=\operatorname{diag}\left(\lambda_{l, 1}, \cdots, \lambda_{l, s}\right), \lambda_{l, i}$ 's are real numbers, $1 \leq i \leq s, 0 \leq l \leq$ $k-1$ such that $P_{l}=Q \Lambda_{l} Q^{T}, 0 \leq l \leq k-1$, then $X=Q \Lambda Q^{T}$ is a solution

of (25), where $\Lambda=\operatorname{diag}\left(\lambda_{1}, \cdots, \lambda_{s}\right)$ and $\lambda_{i}$ is the non-negative solution of

$$
\lambda_{i}=\sum_{l=0}^{k-1}\left(\lambda_{l, i}^{2} \lambda_{i}\right)^{\alpha_{l}}, 1 \leq i \leq s,
$$

where $\lambda_{i}>0$ if $\sum_{l=0}^{k-1}\left|\lambda_{l, i}\right|^{2 \alpha_{l}}>0$, and $\lambda_{i}=0$ if $\sum_{l=0}^{k-1}\left|\lambda_{l, i}\right|^{2 \alpha_{l}}=0$.

Theorem 4.5 If $C=\sum_{l=0}^{k-1}\left(P_{l}^{T} P_{l}\right)^{\alpha_{l}}$ is positive definite, then for arbitrary initial positive definite matrices $X_{i}>0,0 \leq i \leq k-1$, the matrix sequence $\left\{X_{i}: i \geq 1\right\}$ defined by (26) converges to the unique positive definite matrix solution of (25), independent of the initial matrices $X_{i}>0,0 \leq i \leq k-1$. 
Acknowledgement The authors sincerely thank the referees for their valuable suggestions and comments.

This paper is partly funded by ARL (DAAD17-01-P-1204), ARO (DAAD1901-1-0738), NKBRSF on Mathematical Mechanics(G1998030600), the National Natural Science Foundation of China(1999014115), the Outstanding Young Teacher Foundation of Educational Department of China, and the Doctoral Program Foundation of Educational Department of China(60073038).

Xiquan $\mathrm{Shi}^{\dagger}$, Fengshan Liu, Hanson Umoh, Frank Gibson

Department of Mathematics

Delaware State University

Dover, DE 19901

fliu@dsc.edu

$\dagger$ This author is also a professor of Dalian University of Technology, China.

\section{References}

[1] Adi Ben-Isreal and Thomas N.E. Greville, Generalized Inverses: Theory and Applications, John Wiley\&Sons, Inc., 1974.

[2] H.T. Freidtag, Some Stray Footnotes in the Spirit of Recreational Mathematics, Abstracts Amer. Math. Soc. 12.4 (1991): 8.

[3] R.A. Horn and C.R. Johnson. Topics in Matrix Analysis. Cambridge University Press, 1991.

[4] S. Shi, Concerning the Recursive Sequence $A_{n+k}=\sum_{i=1}^{k} a_{i} A_{n+i-1}^{\alpha_{i}}$, The Fibonacci Quarterly, 33 (1995), 240-243.

[5] E. Zeidler, Nonlinear Analysis and its Applications IV, Applications to Mathematical Physics, Springer-Verlag New York Inc., 1985. 